\title{
Ambient temperature effect on Amorphous Silicon (A-Si) Photovoltaic module using sensing technology
}

\begin{abstract}
Temperature and solar irradiance are the two dominating cardinals that determine the electrical performance of Photovoltaic (PV) module. In this paper, an experiment is conducted considering Amorphous Silicon (A-Si) PV module in both indoor and outdoor condition to investigate the temperature effect on A-Si module's performance in terms of efficiency and output power through an automatic resistor selection system. The experimental result shows that A-Si PV module has small temperature coefficient effect; however it has higher effect on solar radiation coefficient. A comparison analysis is evaluated with different models to validate the experimental data.
\end{abstract}

Keyword: Amorphous silicon; Photovoltaic module efficiency; Temperature effect; Module operating temperature 